\title{
Angiotensin receptor neprilysin inhibitor treatment is safe and potentially efficacious in end-stage hypertrophic cardiomyopathy
}

To the Editor Hypertrophic cardiomyopathy (HCM) is an often benign and oligosymptomatic or even asymptomatic condition with normal life expectancy. ${ }^{1,2}$ On the other hand, some patients are characterized by adverse clinical profiles and life-threating complications. Those profiles can be broadly divided into left ventricular (LV) outflow tract obstruction, myocardial ischemia and angina, atrial fibrillation and stroke, ventricular arrhythmias and sudden cardiac death, and last but not least heart failure (HF). ${ }^{1}$

In a minority of patients with HCM, the initially small hypertrophied and hypercontractile LV over time comes to display a dilated, thin-walled, and hypocontractile phenotype. ${ }^{1}$ This phase, called end-stage, burnout, or hypokinetic-dilated, is characterized by progressive and usually severe HF symptoms. ${ }^{1,2}$ Despite the absence of randomized controlled trials, drug management typically includes $\beta$-blockers and multilevel inhibition of the renin-angiotensin-aldosterone system (RAAS) including angiotensin-converting enzyme inhibitors (ACEIs), angiotensin receptor blockers (ARBs), and mineralocorticoid receptor antagonists (MRAs), alongside loop diuretics and digoxin..$^{1,3}$ In patients with symptomatic $\mathrm{HF}$ and broad QRS complex, cardiac resynchronization therapy (CRT) was found to be beneficial, both for improvement of symptoms and prognosis. ${ }^{4}$ Small studies have shown the moderate benefits of CRT in a subset of patients with typical left bundle branch block and LV ejection fraction (EF) of less than $50 \% .{ }^{5}$ Orthotopic heart transplantation (HTx) is an established mode of treatment in severe cases of end-stage HCM; however, due to a shortage of eligible organs, this mode of treatment is restricted. Moreover, graft rejection due to acute cellular reaction, susceptibility to infection caused by prolonged immunosuppression, vasculopathy, and neoplasms substantially limit long-term effects of HTx. ${ }^{6}$ The advent of mechanical LV assist devices (LVADs) is improving the poor prognosis of the majority of patients with severe HF. Still, patients with end-stage HCM are not good candidates for LVAD therapy owing to their relatively small LV cavity sizes. ${ }^{1}$

Neprilysin (NEP) is a membrane-bound, zinc-dependent endopeptidase that cleaves peptides with a molecular weight below $3000 \mathrm{Da} .{ }^{7} \mathrm{NEP}$ is not only a ubiquitous enzyme, as it has been found in the brain, heart, lungs, peripheral circulation, adrenal glands, thyroid gland, gastrointestinal tract, and arthritic synovium, but it also has an important enzymatic role in numerous biological reactions. ${ }^{7}$ So far, NEP is best known for its activity in the cardiovascular system where it degrades natriuretic peptides (NPs), angiotensins, bradykinin, endothelin, and adrenomedullin. ${ }^{8}$ However, NEP is also involved in the nervous system, where it acts on endorphins, enkephalins, oxytocin, and neurokinin; in the gastrointestinal system, where it degrades gastrin-releasing peptide, gastric inhibitory peptides, and cholecystokinin; in the respiratory system, where it acts on substance $\mathrm{P}$ and other tachykinins; or in cleaving metabolic peptides, such as glucagon, glucagon-like peptide, $\beta$-lipotropin, and somatostatin. ${ }^{7,9}$ In healthy individuals, but more importantly in those with HF, atrial, B-type, and C-type NPs (ANP, BNP, and CNP, respectively) act via NP receptors to exert largely beneficial effects, such as natriuresis, diuresis, vasodilatation, and suppression of the RAAS and sympathetic nervous system. ${ }^{10}$ NEP is responsible for the proteolytic cleavage of NPs, and its bioactivity is considerably enhanced in $\mathrm{HF}^{9}{ }^{9}$ Of note, avidity of NEP for a particular NP is not identical: it is the highest for CNP and ANP, while BNP is relatively resistant to NEP. ${ }^{9}$ Thus, inhibition of NEP (NEPi) should increase the level of NPs and bradykinins (although the beneficial role of bradykinin in HF is less well documented), which are endogenous, protective agents countering the negative effects of persistent neurohormonal overactivation in HF.

The value of the NEPi has been tested for over 20 years. However, the initial results were not satisfactory because sole NEPi (monotherapy) lacked clinical efficacy. ${ }^{11}$ Therefore, newer agents 
that combine NEPi and anti-RAAS activity via either anti-ACEI or ARB activity, called vasopeptidase inhibitors, have been developed and tested in HF. ${ }^{9}$ In the medium-sized IMPRESS study, ${ }^{12}$ omapatrilat (vasopeptidase inhibitor) significantly reduced the rates of composite endpoint (death and HF hospitalization) compared with lisinopril (ACEI) alone. However, in the much larger OVERTURE study, ${ }^{13}$ omapatrilat was not superior to enalapril (ACEI) alone in reducing the risk of primary clinical event. Furthermore, any clinical benefit of omapatrilat was overshadowed by the significant increase of adverse effects, including severe angioedema and hypotension..$^{13}$ Therefore, the combination of NEPi and ACEI was abandoned due to lack of safety.

In the recent years, a novel class of agents acting on the RAAS and NEPi, namely, angiotensin receptor neprilysin inhibitors (ARNIs), has shown robust evidence for safety and clinical efficacy. PARADIGM-HF ${ }^{14}$ has been so far the largest study ( $\mathrm{n}=8442)$ on HF that tested the novel combination of sacubitril (NEPi) and valsartan (ARB) versus enalapril (ACEI) in symptomatic patients with $\mathrm{HF}$ and reduced $\mathrm{EF}$ due to ischemic or dilated cardiomyopathy. Importantly, the study was prematurely terminated due to overwhelming benefit of ARNI. ARNI was clearly superior to enalapril in improving prognosis (hazard ratio for all-cause mortality, 0.84; 95\% confidence interval, $0.76-0.93 ; P<0.001$ ), reducing the number of hospitalizations for HF and improving symptoms and physical capacity. ${ }^{14}$

Despite millions invested in the new chemical compounds and numerous clinical trials in the field of HF, ARNI is one of the very few drugs that have recently been actually confirmed to be safe and effective. Thus, the European Society of Cardiology as well as the American College of Cardiology and American Heart Association promptly recognized the new drug in the HF armamentarium and independently developed new recommendations for incorporation of ARNI into HF management. Although minimal differences can be noted within original texts of the guidelines, both Americans and Europeans recommend ARNI (class I B) as a replacement for an ACEI (or ARB) for ambulatory patients with $\mathrm{HF}$ and those with reduced $\mathrm{EF}$ who remain symptomatic despite optimal treatment. ${ }^{3,15}$ To date, the application of ARNI has not yet been reported in end-stage HCM. Thus, we would like to share a first-in-human experience of ARNI treatment in a middle-aged woman with end-stage HCM and overt HF.

The case described here is that of a 43-year old woman diagnosed with nonobstructive HCM 12 years earlier, following a first-ever out-of-hospital sudden cardiac arrest and successful reanimation. Upon discharge, she had been implanted with a cardioverter-defibrillator. An initial echocardiogram had revealed a small LV cavity, moderately hypertrophied basal and mid-segments of the septum, and normal systolic function. The next 10 years were uneventful. Two years before presentation, she began to notice breathlessness on exertion. An echocardiogram showed a mildly enlarged LV cavity and an EF of $40 \%$. Thus, the transition to end-stage phase was diagnosed. Therapy with a $\beta$-blocker, ACEI, and MRA was initiated. Due to a narrow QRS complex, she was not referred for CRT. Despite treatment, her functional performance declined steadily. Eight months later, she was hospitalized due to severe dyspnea. On presentation, she showed left- and right-sided congestive HF (New York Heart Association functional class IV). Her N-terminal pro-B-type natriuretic peptide (NT-proBNP) level was $845 \mathrm{pg} / \mathrm{ml}$ $[<125]$. On echocardiogram, the LV cavity was enlarged (end-diastolic diameter, $62 \mathrm{~mm}$ ) and EF was $30 \%$. At first she was decongested with a moderate dose of loop diuretics. After a few days, a 6-minute walk test was performed, which revealed significant impairment of exercise tolerance (distance, 320 meters; Borg scale, 7). Based on emerging symptoms, gradual LV deterioration despite conventional treatment, and poor prognosis, the patient was scheduled for elective HTx. LVAD therapy was excluded by the surgeons due to a relatively small LV cavity, which could interfere with the device.

Despite a lack of previous reports, we decided on an extraordinary approach, and after the patient's consent, we put her on a trial treatment with ARNI. After a 24-hour washout period from ACEI, we initiated the smallest dose of ARNI (Entresto, Novartis, 24/26 mg, twice daily). She tolerated the drug well with no signs of hypotension. One month later, she reported feeling minimally better. Three months later, she reported a marked improvement in symptoms and exercise tolerance. A 6-minute walk test revealed an increase of distance to 410 meters with a Borg scale of 3 . On echocardiogram, a reduction of end-diastolic diameter to $58 \mathrm{~mm}$ and $\mathrm{EF}$ to $35 \%$ was observed. Moreover, the NT-proBNP level decreased to $248 \mathrm{pg} / \mathrm{ml}$. The patient did not complain of orthostatic hypotension or any other drug-related untoward effects. Therefore, the decision was made to uptitrate the dose of ARNI to $49 / 51 \mathrm{mg}$, twice daily. Due to substantial improvement, the HTx has been temporarily suspended, while the patient remained under close monitoring.

As far as we are aware, ARNI has not been used in patients with end-stage HCM before. Clearly, the results of this 3-month ARNI treatment presented here need to be interpreted with caution; still, we showed that ARNI was safe and moderately efficacious, at least in this case. Therefore, our preliminary results may show promise for patients with end-stage HCM. We believe that there is clearly a case to be made for further randomized studies in this patient group. However, such studies will be relatively difficult to undertake due to a low number of eligible patients, even at referral centers. 
Author names and affiliations Paweł Rubiś, Sylwia Wiśniowska-Śmiałek, Katarzyna Holcman, Agata Leśniak-Sobelga, Magdalena Kostkiewicz, Piotr Podolec (PR, SW-Ś, KH, AL-S, MK, PP: Department of Cardiac and Vascular Diseases, John Paul II Hospital, Kraków, Poland; MK, PP: Jagiellonian University, Medical College, Institute of Cardiology, Kraków, Poland)

Corresponding author Paweł Rubiś, MD, Klinika Chorób Serca i Naczyń, Krakowski Szpital Specjalistyczny im. Jana Pawła II, ul. Prądnicka 80, 31-202 Kraków, Poland, phone: +48 1261422 87, e-mail: pawelrub@poczta.onet.pl

Conflict of interest The authors declare no conflict of interest.

How to cite Rubiś P, Wiśniowska-Śmiałek S, Holcman $\mathrm{K}$, et al. Angiotensin receptor neprilysin inhibitor treatment is safe and potentially efficacious in end-stage hypertrophic cardiomyopathy. Pol Arch Intern Med. 2017; 127 (3): 216-218. doi:10.20452/pamw.3982

\section{REFERENCES}

1 Elliott PM, Anastasakis A, Borger MA, et al. 2014 ESC Guidelines on diagnosis and management of hypertrophic cardiomyopathy: the Task Force for the Diagnosis and Management of Hypertrophic Cardiomyopathy of the European Society of Cardiology (ESC). Eur Heart J. 2014; 35: 2733-2779.

2 Andersson B, Arbustini E, Bilinska Z, et al. Classification of the cardio myopathies: a position statement from the European Society Of Cardiology Working Group on Myocardial and Pericardial Diseases. Eur Heart J. 2008; 29: $270-276$

3 Ponikowski P, Voors AA, Anker SD, et al. 2016 ESC Guidelines for the diagnosis and treatment of acute and chronic heart failure: The Task Force for the diagnosis and treatment of acute and chronic heart failure of the European Society of Cardiology (ESC) Developed with the special contribution of the Heart Failure Association (HFA) of the ESC. Eur Heart J. 2016; 37: $2129-2200$

4 Zareba W, Zareba KM. Which patients with chronic heart failure should be referred for CRT-D implantation? Practical implications of current clinical research. Pol Arch Med Wewn. 2010; 120: 95-102.

5 Rogers DP, Marazia S, Chow AW, et al. Effect of biventricular pacing on symptoms and cardiac remodeling in patients with end-stage hypertrophic cardiomyopathy. Eur J Heart Fail. 2008; 10: 507-513.

6 Antończyk K, Szulik M, Zakliczyński M, et al. Recurrent asymptomatic acute cellular rejection after heart transplantation: monitoring with speckle-tracking echocardiography. Pol Arch Med Wewn. 2016; 126: 700-703.

7 Pankow K, Schwiebs A, Becker M, et al. Structural substrate conditions required for neutral endopeptidase-mediated natriuretic peptide degradation. J Mol Biol. 2009; 393: 496-503.

8 Cruden NL, Fox KA, Ludlam CA, et al. Neutral endopeptidase inhibition augments vascular actions of bradykinin in patients treated with angiotensin-converting enzyme inhibition. Hypertension. 2004; 44: 913-918.

9 Bayes-Genis A, Barallat J, Richards AM. A test in context: neprilysin: function, inhibition, and biomarker. J Am Coll Cardiol. 2016; 68: 639-653.

10 Potter LR. Natriuretic peptide metabolism, clearance and degradation. FEBS J. 2011; 278: 1808-1817

11 Kromer EP, Elsner D, Kahles HW, et al. Effects of atriopeptidase inhibitor UK 79300 on left ventricular hydraulic load in patients with congestive heart failure. Am J Hypertens. 1991; 4: 460-463.

12 Rouleau JL, Pfeffer MA, Stewart DJ, et al. Comparison of vasopeptidase inhibitor, omapatrilat, and lisinopril on exercise tolerance and morbidity in patients with heart failure: IMPRESS randomised trial. Lancet. 2000; 356: $615-620$.

13 Packer M, Califf RM, Konstam MA, et al. Comparison of omapatrilat and enalapril in patients with chronic heart failure: the Omapatrilat Versus Enalapril Randomized Trial of Utility in Reducing Events (OVERTURE). Circulation. 2002; 106: 920-926.

14 McMurray JJ, Packer M, Desai AS, et al. Angiotensin-neprilysin inhibition versus enalapril in heart failure. N Engl J Med. 2014; 371: 993-1004.

15 Yancy CW, Jessup M, Bozkurt B, et al. 2016 ACC/AHA/HFSA Focused Update on New Pharmacological Therapy for Heart Failure: An Update of the 2013 ACCF/AHA Guideline for the Management of Heart Failure:
A Report of the American College of Cardiology/American Heart Association Task Force on Clinical Practice Guidelines and the Heart Failure Society of America. Circulation. 2016; 134: e282-e293. 